\title{
Monte Carlo Simulation Approach to Internal Partition Functions for van der Waals Molecules
}

\author{
A. Riganelli, W. Wang, and A. J. C. Varandas* \\ Departamento de Química, Universidade de Coimbra P-3049 Coimbra Codex, Portugal
}

Received: May 5, 1999; In Final Form: June 25, 1999

\begin{abstract}
Classical Monte Carlo simulation methods have been used to evaluate the internal partition function of diatomic and triatomic van der Waals molecules. All simulation methods are simple to implement and are shown to yield very accurate results for $\mathrm{Ar} \cdots \mathrm{O}, \mathrm{Ar} \cdots \mathrm{O}_{2}$, and $\mathrm{Ar} \cdots \mathrm{CN}$ when compared with the corresponding exact quantum mechanical results. Their efficiencies are also examined.
\end{abstract}

\section{Introduction}

The calculation of the internal partition function $Q_{\text {int }}$, and the density of states $\mathrm{N}$, is an important topic which has a vast range of applications. In fact, its accurate determination for a nonseparable potential is a long standing problem which goes back to the foundations of statistical mechanics. The density of states is required for the evaluation of reaction rate constants in transition state theory (conventional or variational), while the calculation of $Q_{\text {int }}$ is important, e.g., to study the equilibrium properties of bound systems. In turn, its temperature dependence is necessary to evaluate various thermodynamic quantities, such as the Gibbs enthalpy and the Helmholtz function, which are used as standard input for many models in astrophysics; see ref 1 . For example, the solution of the equation of state for cool stellar atmospheres requires very accurate values of $Q_{\text {int. }}{ }^{2,3}$ However, the calculation of $Q_{\text {int }}$ becomes particularly difficult for systems involving weakly bound states such as van der Waals (vdW) molecules. Indeed, the application of approximate methods like the harmonic oscillator and rigid rotor $(\mathrm{HO}+\mathrm{RR})$ approximation (which has been widely used to build up the $\mathrm{JANAF}^{4}$ tables of thermodynamic functions) is expected to give poor results for such floppy (anharmonic) molecules.

Recently, we have been interested in the study of thermolecular association reactions of the general type

$$
\mathrm{A}+\mathrm{BC}+\mathrm{M} \rightarrow \mathrm{ABC}+\mathrm{M}
$$

As prototypes, we have investigated ${ }^{5}$ the recombination reactions for formation of the hydroperoxyl radical and ozone, i.e.

$$
\mathrm{X}+\mathrm{O}_{2}+\mathrm{M} \rightarrow \mathrm{XO}_{2}+\mathrm{M}(\mathrm{X}=\mathrm{H}, \mathrm{O})
$$

where $\mathrm{M}$ is a third body (this will be considered in this work to be an argon atom), and most recently for the reaction ${ }^{6}$

$$
\mathrm{H}+\mathrm{CN}+\mathrm{M} \rightarrow \mathrm{HCN}+\mathrm{M}
$$

The reaction leading to ozone formation is well-known to play an important role in chemistry of the upper atmosphere, particulary in relation to the ozone depletion problem. It comes therefore as no surprise that several previous studies have been reported for such a reaction. On the other hand, the reaction yielding hydrogen cyanide has been the subject of a very detailed study by one of us $^{6}$ with a view to illustrate the range of validity of the various mechanisms and thereby shed light on the possibility of observing direct three-body collisions as argued by Pack and co-workers. ${ }^{7,8}$

Several schemes have been proposed to explain the kinetics of a recombination reaction, namely, the energy-transfer ${ }^{9}$ (ET) mechanism, a mechanism involving excited electronic states ${ }^{10}$ (EE), and the radical-complex or Chaperon ${ }^{5}$ (RC) mechanism (see also ref 11 and references therein). For the purpose of this work, we will focus our attention on the RC mechanism. When applied to the general reaction 1, it involves the following steps:

$$
\begin{gathered}
\mathrm{A}+\mathrm{M} \stackrel{K_{2}}{\rightleftharpoons} \mathrm{M} \cdots \mathrm{A} \\
\mathrm{BC}+\mathrm{M} \stackrel{K_{3}}{\rightleftharpoons} \mathrm{M} \cdots \mathrm{BC} \\
\mathrm{M} \cdots \mathrm{A}+\mathrm{BC} \stackrel{k_{4}}{\longrightarrow} \mathrm{ABC}+\mathrm{M} \\
\mathrm{A}+\mathrm{M} \cdots \mathrm{BC} \stackrel{k_{5}}{\longrightarrow} \mathrm{ABC}+\mathrm{M} \\
\mathrm{M} \cdots \mathrm{A}+\mathrm{M} \cdots \mathrm{BC} \stackrel{k_{6}}{\longrightarrow} \mathrm{ABC}+2 \mathrm{M}
\end{gathered}
$$

Note that according to this mechanism, the low pressure limit of the second-order rate constant for formation of the stable triatomic molecule $\left(\mathrm{HO}_{2}, \mathrm{O}_{3}\right.$, or $\left.\mathrm{HCN}\right)$ assumes the form ${ }^{10}$

$$
k_{0}=\left(K_{2} k_{4}+K_{3} k_{5}\right)[\mathrm{M}]
$$

Thus, an accurate evaluation of the equilibrium constants for processes 4 and 5 is essential to obtain $k_{0}$ as a function of temperature. In turn, the evaluation of the $K_{2}$ and $K_{3}$ equilibrium constants requires the knowledge of $\mathrm{Q}_{\text {int }}$ for the involved vdW

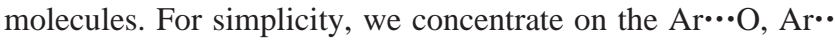
$\cdot \mathrm{O}_{2}$, and $\mathrm{Ar} \cdots(\mathrm{CN}) \mathrm{vdW}$ molecules and omit heretofore the ". .." symbol.

In quantum statistical mechanics (QSM), the internal partition function is represented by the following summation

$$
\mathrm{Q}_{\mathrm{int}}=\sum_{v j}(2 j+1) \exp \left(\beta \epsilon_{v j}\right)
$$

where $\epsilon_{v j}$ is the energy associated with the quantum state $(v, j)$, and the factor $(2 j+1)$ accounts for the rotational degeneracy. Note that eq 10 refers to a specific electronic state of the molecule, and hence, it is assumed that no electronic excited states are involved at the temperatures of interest. In other words, 
the internal partition function assumes the form ${ }^{12}$

$$
\mathrm{Q}_{\text {int }}=\mathrm{Q}_{\mathrm{vr}} \mathrm{Q}_{\mathrm{e}}
$$

with the electronic partition function being $Q_{\mathrm{e}}=1 ; \mathrm{Q}_{\mathrm{vr}}$ stands as usual for vibrational-rotational partition function. For the case of a diatomic molecule, the quantum solution of eq 10 can be obtained by calculating the vibrational-rotational quantum states. However, for a larger system, such a calculation becomes very difficult if not impossible. ${ }^{35}$ Although enormous progress in computer technology has made feasible such vibrationalrotational calculations, the problem of very large CPU times remains. We therefore focus our attention in this work on the calculation of the partition function using classical statistical mechanics (CSM).

The details are organized as follows. In section 2, we show, by comparing the classical solutions with the exact quantum ones, that CSM leads to negligible errors in the case of a diatomic molecule. Then, section 3 presents several Monte Carlo (MC) simulation approaches to the partition functions of the ArO diatomic vdW molecule (section 3.1) and triatomics $\mathrm{ArO}_{2}$ and $\mathrm{ArCN}$ (section 3.2). The conclusions are in section 4.

\section{Classical Approaches to the Partition Function}

For a system composed of $N$ particles confined to a volume $V$, the classical expression for $Q_{\text {int }}$ is

$$
\mathrm{Q}_{\text {int }}=\frac{1}{h^{f}} \iint \cdots \int_{\mathrm{B}} \exp [\beta H(\mathbf{q}, \mathbf{p})] \mathrm{d} \mathbf{q} \mathrm{d} \mathbf{p}
$$

where $f$ is the number of internal degrees of freedom, $H(\mathbf{p}, \mathbf{q})$ is the classical Hamiltonian, $\mathbf{q} \equiv\left\{q_{1}, q_{2}, \ldots, q_{f}\right\}$ are generalized coordinates, $\mathbf{p} \equiv\left\{p_{1}, p_{2}, \ldots, p_{f}\right\}$ the corresponding conjugate momenta, and $\beta=-1 / k T$; the subscript " $B$ " in the last integral indicates that the integrations are only over the degrees of freedom corresponding to a bound system. For $N$ atoms, the total number of degrees of freedom is $3 N$, and hence the above integration are over the $f=3 N-3$ internal degrees of freedom. Note that by removing the coordinates of the center-of-mass (thus, discarding the kinetic energy part due to translation of the entire system), the allowed motions will be confined to regions of phase space corresponding to a bound system. Note also that this requirement depends on the system under consideration, as it will be discussed in the following sections. As usual, $h^{f}$ represents the volume of phase space corresponding to a quantum state (cell).

For a diatomic molecule, the standard way to evaluate the integral in eq 12 consists of adopting a system of spherical polar coordinates $(r, \theta, \phi)$; see Figure 1a. One then gets for the corresponding Hamiltonian

$$
H(\mathbf{q}, \mathbf{p})=\frac{1}{2 I}\left(p_{\theta}^{2}+\frac{p_{\phi}^{2}}{\sin ^{2} \theta}\right)+\frac{p_{r}^{2}}{2 \mu}+V(r)
$$

where $I$ and $\mu$ are the momentum of inertia and the reduced mass of the system, respectively. Thus, the internal partition function is given by

$$
\begin{aligned}
& Q_{\text {int }}=\frac{1}{h^{3}} \int_{0}^{\infty} \int_{0}^{\pi} \int_{0}^{2 \pi} \int_{-\infty}^{\infty} \int_{-\infty}^{\infty} \int_{-\infty}^{\infty} \exp \\
&(\beta H) \mathrm{d} \theta \mathrm{d} \phi \mathrm{d} r \mathrm{~d} p \theta \mathrm{d} p_{\phi} \mathrm{d} p_{\mathrm{r}}
\end{aligned}
$$

where the limits of integration for the variables $\mathbf{q}$ and $\mathbf{p}$ (now $\{r, \theta, \phi\}$ and $\left.\left\{p_{r}, p_{\theta}, p_{\phi}\right\}\right)$ are fixed from the condition that the kinetic energy contribution to the Hamiltonian cannot exceed
TABLE 1: Equilibrium Constant for $\mathrm{Ar}+\mathrm{O} \rightleftharpoons \mathrm{ArO}$ (in $\mathrm{cm}^{3}$ molecule ${ }^{-1}$ ) Calculated from Two Different Potential Energy Curves Using the Methods Described in the Text

\begin{tabular}{rccccc}
\hline & \multicolumn{2}{c}{ EHFACE2 } & & \multicolumn{2}{c}{ LJ(6-12) } \\
\cline { 2 - 3 } \cline { 5 - 6 }$T / \mathrm{K}$ & classical & quantum & & classical & quantum \\
\hline 100 & $1.539 \times 10^{-22}$ & $1.450 \times 10^{-22}$ & & $1.648 \times 10^{-22}$ & $1.607 \times 10^{-22}$ \\
300 & $2.493 \times 10^{-23}$ & $2.352 \times 10^{-23}$ & & $2.682 \times 10^{-23}$ & $2.632 \times 10^{-23}$ \\
500 & $1.122 \times 10^{-23}$ & $1.058 \times 10^{-23}$ & & $1.208 \times 10^{-23}$ & $1.187 \times 10^{-23}$ \\
1000 & $3.875 \times 10^{-24}$ & $3.654 \times 10^{-24}$ & & $4.173 \times 10^{-24}$ & $4.103 \times 10^{-24}$
\end{tabular}

in magnitude that of the potential energy part (we assume the zero of potential energy to correspond to the separated atoms limit). By integrating over the angles, one gets ${ }^{14-16}$ after some algebraic manipulation

$$
\mathrm{Q}_{\mathrm{int}}=\frac{8 \pi^{2}}{h^{3}}(2 \mu k T)^{3 / 2} \int_{\sigma}^{\infty} \exp [\beta V(r)] \gamma[3 / 2, \beta V(r)] r^{2} \mathrm{~d} r
$$

where $\gamma(a, b)$ is the incomplete $\gamma$ function, ${ }^{17}$ and $\sigma$ is the distance for which $V(\sigma)=0$. The integral in eq 15 is a one-dimensional integral and can be resolved easily by any conventional numerical method. For some diatomic potential models such as the generalized Lennard-Jones $(m, n)$, Morse, and Woolley curves, it is even possible to get the analytical solution of eq $15 . .^{18,19}$

To investigate the validity of the classical approach, we first compare the diatomic results for the internal partition function obtained from eq 15 with their accurate quantum analogs. For this purpose, we have calculated $Q_{\text {int }}$ in eq 15 for ArO using two different models: EHFACE2 ${ }^{20}$ (see also ref 21) and Lennard-Jones $(6-12)$ potential functions (the latter is defined by $^{22} R_{\min }=6.65 a_{0}$ and $\epsilon=-0.0002798 E_{h}$ ). In turn, the exact quantum value of $Q_{\text {int }}$ has been obtained from eq. 10 with the $\epsilon_{v j}$ calculated by solving numerically the $1 D$ nuclear Schrödinger equation using the standard Numerov-Cooley algorithm. ${ }^{23,24}$ Classically, the integration of eq 15 for the EHFACE2 potential function has been carried out using the trapezoidal method, while for the Lennard-Jones (6-12) potential function, we have used the analytical solution proposed by Guérin. ${ }^{18,19}$ From the calculated $\mathrm{Q}_{\text {int }}$ for $\mathrm{ArO}$, we have then determined the equilibrium constant $\left(K_{\text {eq }} \equiv K_{2}\right)$ for reaction 4 by applying the standard formalism of statistical mechanics. ${ }^{25}$ Table 1 shows the results obtained from both the CSM and QSM approaches using the above two potential energy curves over a wide range of temperatures. Clearly, the agreement between the CSM and QSM results are very good for a given potential. This warrants in principle the use of the CSM simulation approach to compute accurately the partition function of larger polyatomics.

Unfortunately, the CSM approach cannot be formulated analytically for a polyatomic molecule when using a realistic potential energy surface. Indeed, this problem has only been solved (see ref 26) for the case where $\lim _{\mathbf{q} \rightarrow \infty}|V(\mathbf{q})|=\infty$. In particular, a difficulty which arises when formulating the problem analytically refers to the choice of coordinate system. Probably, the natural option for a triatomic molecule would be to use bipolar coordinates. ${ }^{27}$ However, eq 12 cannot be simplified to a one-dimensional integral as in the diatomic case, and hence the viability of the CSM approach for $Q_{\text {int }}$ depends essentially on the efficiency of the numerical method which deals with the multidimensional integral in eq 12. Usually, the Monte Carlo simulation method is applied to multidimensional integrals with good results and, for this purpose, we investigate in the next section how it performs for diatomics and triatomics. 


\section{The Monte Carlo Simulation Method}

3.1. Calculation of $Q$ int for ArO. As usual, the diatomic molecule is described in phase space by the six Cartesian coordinates $q^{(i)}(i=1-6)$ and their associated momenta $p^{(i)}(i$ $=1-6)$. We further define the coordinates such that $Q^{(i)}(i=$ $1-3)$ indicates the position of the center of mass of the molecule, and $Q^{(i)}(i=4-6)$ the position of atom B with respect to atom $\mathrm{A} ; P^{(i)}(i=1-6)$ are the corresponding conjugate momenta. It therefore remains to define the conditions representative of the bound system. Clearly, they correspond to situations where the internal energy is smaller than or equal to the dissociation energy, which implies $H(\mathbf{p}, \mathbf{q}) \leq 0$ where

$$
H(\mathbf{p}, \mathbf{q})=\frac{1}{2 \mu_{i}} \sum_{i, 6} P^{(i)^{2}}+V\left(Q_{4}, Q_{5}, Q_{6}\right)
$$

We emphasize that the reference potential energy corresponds to the infinitely separated atoms and that we have not taken into account any quasi-bound states of the molecule (for a discussion on this topic, see ref 2). Three variants of the Monte Carlo technique have then been investigated to calculate $Q_{\text {int }}$, which are described next.

3.1.1. Crude Monte Carlo Technique. We define here for the six coordinates a minimum and a maximum displacement, namely, $Q_{\min }, Q_{\max }, P_{\min }$, and $P_{\max }$. The simulation has then been carried out by defining

$$
\begin{gathered}
Q^{(i)}=Q_{\text {min }}+\left(Q_{\max }-Q_{\min }\right) \xi \\
P^{(i)}=P_{\text {min }}+\left(P_{\text {max }}-P_{\text {min }}\right) \xi
\end{gathered}
$$

where $\xi$ is a random number in the range $[0-1] ;(i=4,6)$. At this stage the Hamiltonian is calculated and the point accepted in case its value is smaller than zero (bound state situation); otherwise, it is rejected, i.e., $\exp \left(-\beta H_{i}\right)=0$, and a new point sampled. The integral in eq 12 is then replaced by the standard Monte Carlo summation

$$
S=V_{\mathrm{s}} \frac{\sum_{i=1}^{N_{\text {in }}} \exp \left(-\beta H_{i}\right)}{N_{\text {tot }}}
$$

where $N_{\text {tot }}$ is the total number of randomly generated points in phase space, $N_{\text {in }}$ the number of points satisfying the condition $[H(\mathbf{p}, \mathbf{q}) \leq 0]$, and the total sampled volume $V_{\mathrm{s}}$ is defined by

$$
V_{\mathrm{s}}=\prod_{i=4}^{6}\left(Q_{\max }^{(i)}-Q_{\min }^{(i)}\right)\left(P_{\max }^{(i)}-P_{\min }^{(i)}\right)
$$

Of course, a crucial step in this "crude" Monte Carlo scheme is the definition of the phase space volume to be randomly sampled. In other words, the sampled volume should include the true volume but be as close as possible to it. Unfortunately, there is not an obvious way to accomplish this using Cartesian coordinates. The choice of $P_{\min }$ and $P_{\max }$ is suggested by itself: $P_{\max }=\left(2 \mu E_{\mathrm{d}}\right)^{1 / 2}$ and $P_{\min }=-P_{\max }$, where $\mu$ is the reduced mass of the system, and $E_{\mathrm{d}}$ is the dissociation energy of the molecule. On the other hand, the selection of position coordinates is arbitrary in the sense that they can assume any value between $-\infty$ and $+\infty$. For practical purposes, one may fix a priori such a range, say, by considering only distances smaller than $30 a_{0}$ or so since the interaction energy should be essentially negligible outside this range. Such a selection implies $Q_{\text {int }}=$ $-15 a_{0}$ and $Q_{\max }=15 a_{0}$. Table 2 shows the results obtained
TABLE 2: A Comparison of the Classical MC and QSM Results for $Q_{\text {int }}$ Obtained for the ArO vdW Molecule at $T=$ $300 \mathrm{~K}$ Using the EHAFACE2 Potential Energy Curve

\begin{tabular}{llll}
\hline method & \multicolumn{1}{c}{ Qint } & $N_{\text {tot }}$ & $\epsilon(\%)$ \\
\hline $\mathrm{A}^{a}$ & $705.4 \pm 6.8$ & $10^{6}$ & 1.1 \\
$\mathrm{~B}^{b}$ & $701.0 \pm 3.5$ & $10^{6}$ & 3.9 \\
$\mathrm{C}^{c}$ & $699.6 \pm 2.6$ & $10^{6}$ & 100 \\
$\mathrm{QSM}^{d}$ & 699.7 & &
\end{tabular}

${ }^{a}$ Classical crude MC simulation method, this work. ${ }^{b}$ Classical improved MC simulation method, this work. ${ }^{c}$ Classical Barker's method, this work. ${ }^{d}$ Quantum statistical mechanical result, this work.

when these values are adopted to calculate $Q_{\text {int }}$. Clearly, the efficiency of this crude MC method is poor (this is defined by $\epsilon=N_{\text {in }} / N_{\text {tot }}$ ): $\sim 1 \%$. However, this problem can be easily overcome by increasing the number of trials. For example, by using $10^{7}$ trials, the calculated value becomes very close to the QSM result.

As usual, the standard deviation error associated with eq 19 is given by

$$
\sigma=V_{\mathrm{s}} \sqrt{\frac{\left\langle\exp ^{2}\left(-\beta H_{i}\right)\right\rangle-\left\langle\exp \left(-\beta H_{i}\right)\right\rangle^{2}}{N_{\text {tot }}-1}}
$$

where the angle brackets denote taking the arithmetic mean over the $N_{\text {tot }}$ sampling points,

$$
\begin{gathered}
\left\langle\exp ^{2}\left(-\beta H_{i}\right)\right\rangle=\frac{1}{N_{\text {tot }}} \sum_{i=1}^{N_{\text {in }}} \exp ^{2}\left(-\beta H_{\mathrm{i}}\right) \\
\left\langle\exp \left(-\beta H_{i}\right)\right\rangle=\frac{1}{N_{\text {tot }}} \sum_{i=1}^{N_{\text {in }}} \exp \left(-\beta H_{\mathrm{i}}\right)
\end{gathered}
$$

3.1.2. Improved Crude Monte Carlo Technique. Naturally, a simple way to improve the efficiency of the crude Monte Carlo simulation consists of decreasing as much as possible the sampling volume. This can be partially fullfilled by choosing an appropriate coordinate system for phase space sampling. For example, to perform the coordinate space sampling, we first recall that the Cartesian coordinates are related to the polar ones through the relations

$x=r \sin \theta \cos \phi, y=r \sin \theta \sin \phi, \quad$ and $\quad z=r \cos \theta$

with the infinitesimal element of volume being given by

$$
d V=r^{2} \sin \theta \mathrm{d} \theta \mathrm{d} \phi \mathrm{d} r
$$

where the limits are $0 \leq \theta \leq \pi$ and $0 \leq \phi \leq 2 \pi$. Then, we note that the angular part can be integrated analytically, and hence the random sampling is reduced to the variables $r$ and $p$. On the other hand, the volume of a sphere is proportional to the cube of its radius, and hence a uniform sampling procedure in $r$ and $p$ requires that the two variables are obtained from

$$
\begin{aligned}
& r=\left[r_{\text {min }}^{3}+\left(r_{\text {max }}^{3}-r_{\text {min }}^{3}\right) \xi\right]^{1 / 3} \\
& p=\left[p_{\text {min }}^{3}+\left(p_{\text {max }}^{3}-p_{\text {min }}^{3}\right) \xi\right]^{1 / 3}
\end{aligned}
$$

Thus, an advantage of using such a coordinate system is that the sampling volume can now be split on the product of two spherical volumes. One has, 


$$
V_{\text {eff }}=V_{\mathrm{Q}} V_{\mathrm{P}}
$$

where $V_{Q}$ represents the configuration space volume, and $V_{P}$ the corresponding momentum space volume:

$$
\begin{aligned}
& V_{Q}=\frac{4}{3} \pi\left(r_{\max }^{3}-r_{\min }^{3}\right) \\
& V_{P}=\frac{4}{3} \pi\left(p_{\max }^{3}-p_{\min }^{3}\right)
\end{aligned}
$$

Although the definition of $p_{\max }$ is the same as stated above, $p_{\text {min }}$ will now be zero. Concerning the $r$ variable, its range can be chosen such that $r_{\max }$ corresponds to a distance for which the interaction energy is negligible (say, $r_{\max }=15 a_{0}$ ). In turn, $r_{\min }$ must be chosen as small as possible but different from zero (e.g., $r_{\min }=0.01 a_{0}$ ) to avoid an overflow due to the Coulombic pole in the potential energy at the united atom limit of the collapsed diatomic. We still use eq 19 to evaluate the integral in eq 12, and eq 20 for the associated errors. Somewhat surprisingly, the efficiency turns out to be only about 4 times larger than that of the crude MC approach; see Table 2. This can be understood from the fact that the sampling volume has been reduced by a factor of 4 ; note that we have now two spheres, with each of them having a volume nearly two times smaller than that of the cube in which they are inscribed.

3.1.3. Barker's Algorithm. The approach described in this section is an adaptation of a method proposed by Barker ${ }^{28}$ which has originally been suggested to calculate the sum and density of states of a given molecule. As before, the main idea is to choose the sampling volume to be as close as possible to the integrated one such as to increase the efficiency of the method. Of course, in the limit, one may achieve unit efficiency. In the approaches described in the previous paragraphs, each random variable was sampled independently from each other leading to a uniform distribution. In this approach, the range of each variable is instead considered to be a function of the variables previously sampled. Thus, the obtained distribution is no longer uniform, and weight factors need to be considered. For convenience, we report next the algorithm adopted, and provide the final expression to calculate the integral in eq 12; further details and formal demonstrations can be found in the original work. 28

The general procedure consists of the following steps: 1 . Define a boundary surface in the $n$-dimensional space $B=$ $F\left(\zeta^{(1)}, \ldots, \zeta^{(n)}\right)$ such that the integration domain is completely contained in this surface. 2. Fix the range $\left(\xi_{\max }-\xi_{\min }\right)$ for the $\xi^{(1)}$ variable, and the $\zeta_{\min }^{(j)}$ value for all others $(j=2, n) .3$. Sample at random the variable in the given range. 4. Calculate the value of $\xi_{\max }$ according to the boundary surface defined in step 1 for the variables $\xi^{(j)}(j=2, n)$, and repeat step 3 for all these variables. 5. Calculate the weight for each sampled point $g$ according to

$$
W_{g}=\prod_{j=1}^{n}\left[\zeta_{\max }^{(j)}-\xi_{\min }^{(j)}\right]
$$

6. Calculate the integral according to

$$
S=\Sigma f_{g} W_{g} / N
$$

where $f_{g}=\exp (-H(\mathbf{q}, \mathbf{p}) / k T)$, and $N$ is the total number of sampled points. Clearly, we have now $N \equiv N_{\text {tot }} \equiv N_{\text {in }}$, and hence all points satisfy the condition $H \leq 0$.
TABLE 3: Values of $Q_{\text {int }}$ for the ArO vdW Molecule over a Wide Range of Temperatures: See Also the Text and Table 2

\begin{tabular}{rcccc}
\hline & & \multicolumn{3}{c}{ MC } \\
\cline { 3 - 5 }$T / \mathrm{K}$ & $\mathrm{QSM}^{a}$ & method A $^{b}$ & $\operatorname{method~B}$ & Method C $^{d}$ \\
\hline 100 & 461.2 & $463.4 \pm 4.6$ & $462.1 \pm 2.4$ & $461.2 \pm 1.9$ \\
200 & 629.3 & $633.9 \pm 6.1$ & $630.5 \pm 3.2$ & $629.3 \pm 2.4$ \\
400 & 738.0 & $744.4 \pm 7.2$ & $739.4 \pm 3.7$ & $738.0 \pm 2.8$ \\
500 & 762.2 & $768.9 \pm 7.4$ & $763.6 \pm 3.8$ & $762.1 \pm 2.8$ \\
600 & 778.8 & $785.8 \pm 7.6$ & $780.2 \pm 3.9$ & $778.7 \pm 2.9$ \\
700 & 790.9 & $798.0 \pm 7.7$ & $792.3 \pm 4.0$ & $790.8 \pm 2.9$ \\
800 & 800.0 & $807.4 \pm 7.8$ & $801.5 \pm 4.0$ & $800.0 \pm 3.0$ \\
900 & 807.3 & $814.7 \pm 7.8$ & $808.8 \pm 4.0$ & $807.2 \pm 3.0$ \\
1000 & 813.1 & $820.7 \pm 7.9$ & $814.6 \pm 4.1$ & $813.0 \pm 3.0$
\end{tabular}

${ }^{a}$ Quantum statistical mechanical result, this work. ${ }^{b}$ Classical crude MC simulation method, this work. ${ }^{c}$ Classical improved MC simulation method, this work. ${ }^{d}$ Classical Barker's method this work.

For a diatomic, the definition of the boundary surface is given straightforwardly by $H(p, r)=0$, which implies $p^{2} / 2 \mu=-V(r)$. Following the above scheme, we then choose $r_{\min }$ as the value of $\sigma$ in eq 15 and $r_{\max }$ as the distance for which we may consider the interaction energy to be negligible (in this work, we consider $r_{\max }=15 a_{0}$ ). Fixed $p_{\min }=0$, we then choose $r$ at random while the value of $p_{\max }$ is fixed from the boundary surface. The sampling of the other variables (step 3 ) is done next using eqs 24 and 25. Regarding the weight factors, one should keep in mind that we are using spherical coordinates, and hence the weights must be calculated acording to eqs 27 and 28. The standard deviation associated with eq 30 assumes now the form ${ }^{28,29}$

$$
\sigma^{2}=[N(N-1)]^{-1} \Sigma\left(f_{g} w_{g}-S\right)^{2}
$$

Table 2 compares the results from the three sampling methods described above, all based on $10^{5}$ sampling points. Clearly the best agreement with the exact quantum result is obtained with Barker's method. Similarly, for the same degree of precision, the number of required sampling points is smallest for the Barker's approach and largest for the crude MC method. Note that the efficiency of a MC method is usually defined as the percentage of the physically acceptable points (those satisfying the boundary conditions) among the total number of sampled points. Obviously, Barker's method leads always to $100 \%$ efficiency, since all sampled points are physically acceptable. Table 3 shows in turn the results obtained for $Q_{\text {int }}$ over the range of temperatures $100 \leq T / \mathrm{K} \leq 1000$, while providing also a comparison with the values obtained from other methods. As observed, there is good agreement between all results.

3.2. Calculation of $Q_{\text {int }}$ for $\mathrm{ArO}_{2}$ and $\mathrm{ArCN}$. The first difficulty which arises when one applies Barker's procedure to a triatomic system is to define the binding condition, i.e., the region of phase space for which the internal energy is smaller than the dissociation energy $\left(E_{\mathrm{d}}\right)$. Indeed, for a polyatomic molecule, this definition is not unique, since there is more than one dissociation channel. The problem gets simplified in the case of a vdW molecule, since the natural choice will be to assume as reference energy that associated to the atom + bound diatomic dissociation channel, e.g., $\mathrm{ArO}_{2}(\mathrm{CN}) \rightarrow \mathrm{O}_{2}(\mathrm{CN})+\mathrm{Ar}$, and hence, the values of $E_{\mathrm{d}}$ used in this work are for the $\mathrm{ArO}_{2}$ and $\mathrm{ArCN},-122.9 \mathrm{~cm}^{-1}$ and $-58.0 \mathrm{~cm}^{-1}$, respectively.

In a space-fixed Cartesian system of coordinates, a point in phase space will be described by $q_{i}(i=1,9)$ and $p_{i}(i=1,9)$. Since we are interested in the calculation of $Q_{\text {int }}$, we adopt the generalized coordinates described in ref 30 in such a way that $\left(Q_{4}, Q_{5}, Q_{6}\right)$ identify the position of atom $\mathrm{C}$ with respect to 


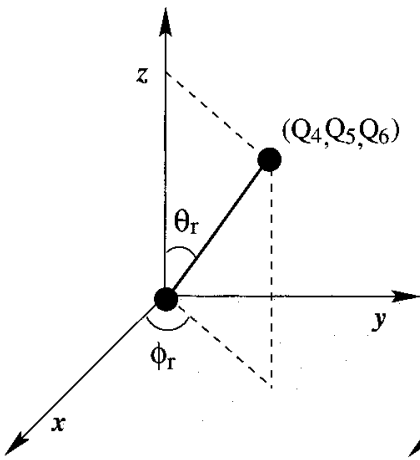

(a)

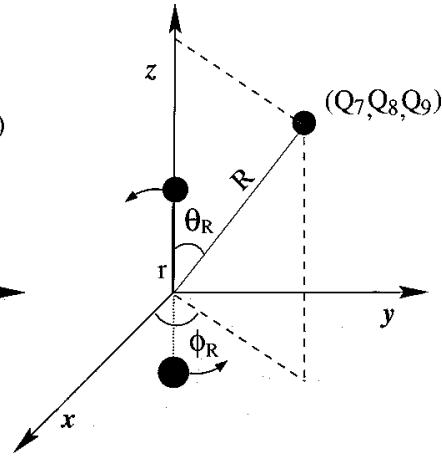

(b)
Figure 1. Transformation between the Cartesian (see the text) and spherical polar coordinate systems: (a) $\left(Q_{4}, Q_{5}, Q_{6}\right) \rightarrow\left(r, \theta_{r}, \phi_{r}\right)$, (b) $\left(Q_{7}, Q_{8}, Q_{9}\right) \rightarrow\left(R, \theta_{R}, \phi_{R}\right)$

atom $\mathrm{B}$, and $\left(Q_{7}, Q_{8}, Q_{9}\right)$ represents the position of atom $\mathrm{A}$ relative to the center of mass of the diatomic $\mathrm{BC}$. In this coordinate system, the classical Hamiltonian for internal motion assumes the form

$$
H=\frac{1}{2 \mu_{\mathrm{BC}}} \sum_{i=4}^{6} P_{i}^{2}+\frac{1}{2 \mu_{\mathrm{A}, \mathrm{BC}}} \sum_{i=7}^{9} P_{i}^{2}+V\left(Q_{4}, \ldots, Q_{9}\right)
$$

while the boundary condition is defined by $H\left(Q_{4}, \ldots, Q_{9}, P_{4}\right.$, $\left.\ldots, P_{9}\right)=E_{d}$. Because we want to compare the results of our simulation with those obtained by solving the $2 D$ quantum problem, we then freeze the diatomic distance $r$ at its equilibrium value $\left(r_{\mathrm{e}}^{\left(\mathrm{O}_{2}\right)}=2.2818 a_{0}\right.$ and $\left.r_{\mathrm{e}}^{(\mathrm{CN})}=2.214 a_{0}\right)$.

When adopting the crude and improved MC methods, the efficiency obtained is in both cases very small $(0.0001 \%)$ which makes their error bars unacceptable. For this reason, we focus on the results obtained by using Barker's algorithm, since the other methods are unlike to be of practical interest for systems of high dimensionality. However, prior to discussing the results, we need to introduce some further technical details.

First, as in the diatomic case, we adopt spherical polar coordinates for both position and momenta to perform the sampling (see Figure 1). The sampling in configuration space for the Jacobi coordinate $R$ is performed using eq 24, while the other Jacobi coordinate $r$ is fixed at its equilibrium value. Since the boundary condition is defined as usual by $H(\mathbf{q}, \mathbf{p})=E_{\mathrm{d}}$ and the range for the $r$ variable has been fixed ( $r$ is in our case held as a constant value), we may follow the general recipe described in the previous subsection. Specifically, the sampling of phase space involves the following steps: 1 . Fix $\theta_{R}$ at its equilibrum value $\left(\theta_{R}=90^{\circ}\right.$ for $\mathrm{ArO}_{2}$, and $\theta_{R}=100^{\circ}$ for $\left.\mathrm{ArCN}\right), P_{r}=0$, and $P_{R}=0$. With $r$ fixed at its equilibrium value, apply the boundary condition to calculate $R_{\max }$ and $R_{\min }$, and then sample $R$. 2. Using the $R$ value obtained from the previous step and $P_{r}$ $=P_{R}=0$, apply the boundary condition to obtain $\theta_{R_{\max }}$ and $\theta_{R_{\min }}$ from which $\theta_{R}$ is then sampled. 3. From $\mathrm{V}\left(r, R, \theta_{R}\right)$ and $P_{R}=0$, obtain $\left.P_{r}^{\max }=2 \mu\left(E_{\mathrm{d}}-V\right)\right]^{1 / 2}$ and sample $P_{r} .4$. Calculate $P_{R}^{\max }$ from $P_{R}^{\max }=\left[2 \mu\left(E_{\mathrm{d}}-V-P_{r}^{2} / 2 \mu\right)\right]^{1 / 2}$, and then sample $P_{R}$. 5. Determine the weight factors as

$$
w_{g}=V_{Q}^{r} V_{Q}^{R} V_{P}^{r} V_{P}^{R}
$$

where

$$
V_{Q}^{r}=4 \pi r^{2}
$$

$$
\begin{gathered}
V_{Q}^{R}=2 \pi R_{\max }^{3}\left[\cos \left(\theta_{R_{\min }}\right)-\cos \left(\theta_{R_{\max }}\right)\right] / 3- \\
2 \pi R_{\min }^{3}\left[\cos \left(\theta_{R_{\max }}\right)-\cos \left(\theta_{R_{\max }}\right)\right] / 3 \\
V_{P}^{r}=\pi P_{r_{\max }^{2}} \\
V_{P}^{R}=4 / 3 \pi P_{R_{\max }}^{2}
\end{gathered}
$$

The results obtained when such a a procedure is applied to the title triatomic systems are reported in Figures 2 and 3. Note that the MC simulation has been performed at selected temperatures, ranging from $\mathrm{T}=100-5000 \mathrm{~K}$, the number of sampling points being $10^{6}$. Figures 2 and 3 also present a comparison between the MC simulation result (obtained by solving the classical phase space integral) and the QSM internal partition functions, which we discuss in the next paragraph.

To obtain the QSM results, we have adopted a quantum reduced-dimensionality approach, and considered the distance in the diatomic fixed at its equilibrium geometry. This should be a good assumption since we are focusing on vdW molecules, for which we have considered realistic double many-body expansion $^{31,32}$ (DMBE) potential energy surface described elsewhere ${ }^{5,33}$ The $2 \mathrm{D}$ problem has then been solved by using the coupled-channels approach as implemented in the BOUND computer code of Hutson. ${ }^{34,35}$ Specifically, the coupled equations have been propagated for the $\mathrm{ArO}_{2}$ molecule between $R_{\min }=$ $2.6 \AA$ and $R_{\max }=14.0 \AA$ with a step size of $d R=0.05 \AA$, while for the rotational constant of $\mathrm{O}_{2}$ we have used $B_{\mathrm{e}}=$ $1.44563 \mathrm{~cm}^{-1} .36$ The total number of calculated vibrationalrotational levels and maximum total angular momentum have been found to be 1380 and $J=36$, respectively. In turn, for ArCN, we have used the results reported elsewhere ${ }^{6}$ using the same methodology. The vibrational-rotational partition functions were then calculated by carrying out the summation in eq 10 with $j$ replaced by the total angular momentum quantum number $J$ of the complex which is obtained by coupling $\left|j M_{j}\right\rangle$ (the total angular momentum quantum number of the diatomic molecule; note that for $\mathrm{O}_{2}$ the Bose-Einstein statistics forbids even rotational states) and $\left|L M_{L}\right\rangle$ (the end-over-end rotations of the complex). ${ }^{37}$ Clearly, the plots in Figures 2 and 3 show that an excellent agreement is obtained for both systems. In fact, for both $\mathrm{ArCN}$ and $\mathrm{ArO}_{2}$, the bottom parts of Figures 2 and 3 indicate that the deviations between the MC simulation and the exact QSM results are always smaller then $1 \%$.

\section{Conclusions}

The modeling of chemical reactions requires a detailed knowledge of the relevant partition functions over a wide range of temperatures. In particular, the reliability of the calculated properties for reactions such as those in eqs 2 and 3 depends critically on the accuracy of the equilibrium constant for formation of the intermediate species involved in the RC mechanism. Since the evaluation of $Q_{\text {int }}$ is nontrivial and the usual approximations (such as $\mathrm{HO}+\mathrm{RR}$, and related models) breakdown in the case of $\mathrm{vdW}$ molecules, the classical MC simulation approach suggested in the present work looks promising. Indeed, in all cases the exact but more cumbersome QSM calculations agree typically with the classical simulation results within a few tenths of a percent or so.

We have also examined the efficiency in several variants of the MC approach, and concluded that Barker's algorithm is to be recommended. It provides a general methodology to solve multidimensional integrals and may find a wide application in 

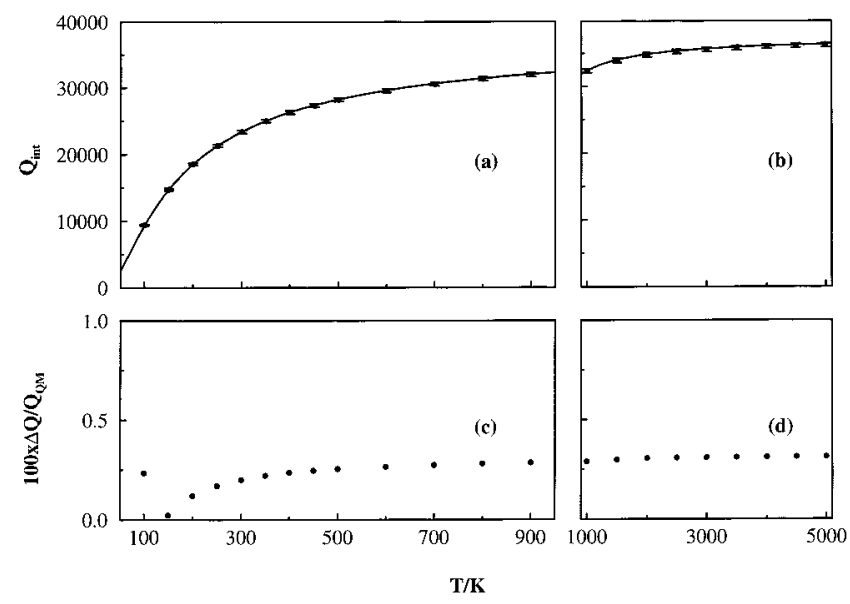

Figure 2. Top panels (a) and (b): $Q_{\text {int }}$ as a function of temperature for the $\mathrm{ArO}_{2} \mathrm{vdW}$ molecule. The solid line represents the exact-quantum result, while the dots indicate the $\mathrm{MC}$ simulation results with errors bars. Bottom parts $\mathrm{c}$ and d: relative error in percent between the QSM and $\mathrm{MC}$ simulation results.

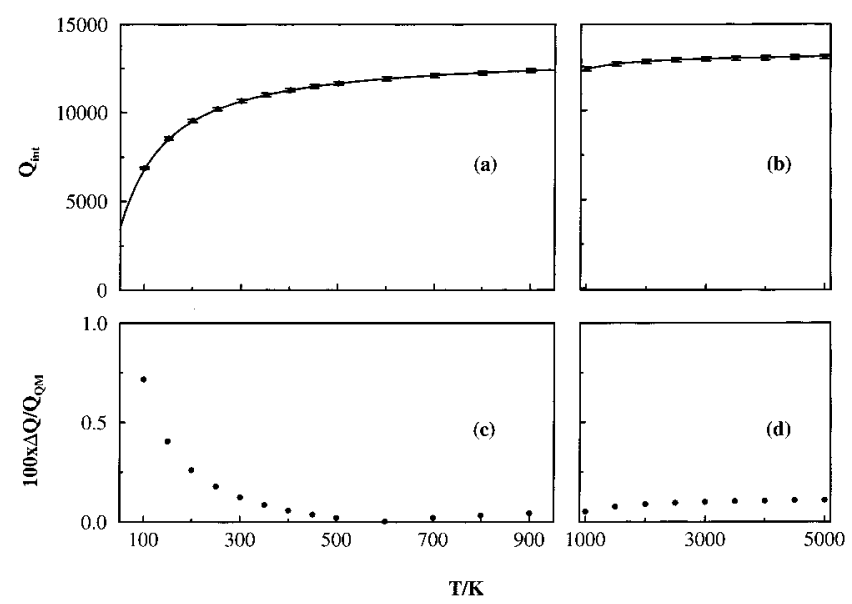

Figure 3. As in Figure 2 but for the $\mathrm{ArCN}$ vdWmolecule.

statistical mechanics where such integrals are frequent. In particular it may possibly be of interest to calculate sums of states for transition states of any looseness, provided that one is able to establish the adequate boundary conditions. Specially notable is the fact that for the title systems the method is simple to implement and the computational effort relatively small. Hopefully, it will be equally useful to handle the general polyatomic case.

Acknowledgment. This work has the support of Fundação para a Ciência e Tecnologia, Portugal, under programmes
PRAXIS XXI and FEDER (Contract 2/2.1/QUI/408/94). It has also benefited from an EC Grant under Contract CHRX-CT 940436.

\section{References and Notes}

(1) Neale, L.; Tennyson, J. Astrophys. J. 1995, 454, L169.

(2) Irwin, A. W. Astron. Astrophys. 1987, 182, 348.

(3) Irwin, A. W. Astron. Astrophys. Suppl. 1988, 74, 145.

(4) Chase Jr., M. W.; Davies, C. A.; Downey, J. R., Jr.; Frurip, D. J.; McDonald, R. A.; Syveraud, A. N. JANAF Thermodynamic Tables, 3rd. ed.; American Chemical Society: Washington, DC, and American Institute for Physics for the National Bureau of Standards: New York, 1985.

(5) Varandas, A. J. C.; Pais, A. A. C. C.; Marques, J. M. C.; Wang, W. Chem. Phys. Lett. 1996, 249, 264.

(6) Rodrigues, S. P. J.; Varandas, A. J. C. J. Phys. Chem. In press.

(7) Pack, R. T.; Walker, R. B.; Kendrick, B. K. J. Chem. Phys. 1998 109, 6701 .

(8) Pack, R. T.; Walker, R. B.; Kendrick, B. K. J. Chem. Phys. 1998, $109,6714$.

(9) Gross, A.; Billing, G. D. Chem. Phys. 1993, 173, 393.

(10) Hippler, H.; Rahn, R.; Troe, J. J. Chem. Phys. 1990, 93, 6560.

(11) Schwenke, D. W. J. Chem. Phys. 1990, 92, 7267.

(12) Harris, G. J.; Viti, S.; Mussa, H. Y.; Tennyson, J. J. Chem. Phys. 1998, 109, 7197.

(13) Tennyson, J. Comput. Phys. Commun. 1986, 42, 257.

(14) Dardi, P. S.; Dahler, J. S. J. Chem. Phys. 1990, 93, 3562.

(15) Hill, T. L. J. Chem. Phys. 1955, 23, 617.

(16) Bunker, D. L.; Davidson, N. J. Am. Chem. Soc. 1958, 80, 5090.

(17) Abramowitz, M.; Stegun, I. A. Handbook of Mathematical Functions; Dover: New York, 1970.

(18) Guérin, H. J. Phys. B: At. Mol. Opt. Phys. 1992, 25, 1697.

(19) Guérin, H. J. Phys. B: At. Mol. Opt. Phys. 1992, 25, 3371.

(20) Varandas, A. J. C.; Silva, J. D. J. Chem. Soc., Faraday Trans. 2 1986, $82,593$.

(21) Varandas, A. J. C.; Silva, J. D. J. Chem. Soc., Faraday Trans. 1992, $88,941$.

(22) Aquilanti, V.; Liuti, G.; Pirani, F.; Vecchiocattivi, F.; Volpi, G. G. J. Chem. Phys. 1976, 65, 4751 .

(23) Cooley, J. W. Math. Comput. 1961, 15, 363.

(24) Johnson, B. R. J. Chem. Phys. 1977, 67, 4086

(25) McQuarrie, D. A. Statistical Mechanics; Harper and Row: New York, 1976

(26) Knyazev, V. D.; Tsing, W. J. Phys. Chem. A 1998, 102, 9167.

(27) Rittger, E. Comput. Phys. Commun. 1992, 67, 412.

(28) Barker, J. R. J. Phys. Chem. 1987, 91, 3849.

(29) Hammersley, J. H.; Handscomb, D. C. Monte Carlo Methods; Chapman and Hall: London, 1964.

(30) Karplus, M.; Porter, R. N.; Sharma, R. D. J. Chem. Phys. 1965, 43,3259 .

(31) Varandas, A. J. C. Adv. Chem. Phys. 1988, 74, 255.

(32) Varandas, A. J. C. Chem. Phys. Lett. 1992, 194, 333.

(33) Rodrigues, S. P. J.; Varandas, A. J. C. J. Phys. Chem. 1998, 102 , 6266.

(34) Hutson, J. M., BOUND computer code, Version 5 (1993); distributed by Collaborative Computational Project No. 6 of the Science and Engineering Research Council, U.K.

(35) Hutson, J. M. Comput. Phys. Commun. 1994, 84, 1.

(36) Huber, K. P.; Herzberg, G. Molecular Spectra and Molecular Structure. IV Constants of Diatomic Molecules; Van Nostrand: New York, 1979.

(37) Tennyson, J.; Avoird, A. V. D. Chem. Phys. Lett. 1984, 49, 105. 